\title{
Reducing plastic zone ahead of crack tips by reshaping stop hole
}

\begin{abstract}
Optimal shape is supposed to remove the damaged material and minimize stresses. In order to validate that, two identical models are created. The first models has a non repaired crack, and the other has a repaired crack with the optimum hole shape Plastic zone size is of crucial importance in this study as it is hypothesized that the hole should be drilled to remove most of this zone. This will leave the rest of the material in virgin state and eliminate any predefined slip planes dislocation.
\end{abstract}

Keywords: optimum hole shape, plastic zone shape
Volume 3 Issue 6 - 2017

Mona Ahmed Awad

Department of Aldelta Academy for Engineering and Technology, Egypt

Correspondence: Mona Ahmed Awad, Department of Aldelta Academy for Engineering and Technology, Production Engineering \& Mechanical Design, Egypt, Email dr.mona7I I@gmail.com

\section{Introduction}

The stop-hole method is a popular emergency repair technique, this method consists of boring a hole in the vicinity of, or centered at the crack tip, to transform the crack into a notch. The ordinary circular stop-hole may not remove the residual stresses associated with the plastic zones which always follow a fatigue crack tip. Plastic deformation will occur at the crack tip as a result of the high stresses that are generated by the sharp stress concentration Irwin's Approach ${ }^{1}$ demonstrates that when yielding occurs, stresses redistribute in order to satisfy equilibrium conditions. To redistribute this excessive force, the plastic zone size must increase Irwin proposed that plasticity makes the crack behave as if it were larger than actual physical size

$$
r_{p}=\frac{1}{\pi}\left(\frac{K_{I}}{\sigma_{y}}\right)^{2}
$$

Where, $r \_p$ is the correct plastic zone size, $\mathrm{K}_{-} \mathrm{I}$ is the stress intensity factor, and $\sigma \_\mathrm{y}$ is the specimen yield stress. Plasticity is based on von Mises and Tresca's yield criteria. ${ }^{2}$ plastic zone shape can be determined as shown in Figure 2 can be obtained as:

Table 1 With angle between specimen symmetry line and the point

$$
r_{p}=\frac{1}{4 \pi}\left(\frac{K_{I}}{\sigma_{Y}}\right)^{2}\left(1+1.5 \sin ^{2} \theta+\cos \theta\right)
$$

\section{Modeling}

The studied model is rectangular plate, with length (h) of $80 \mathrm{~mm}$, width (W) of $40 \mathrm{~mm}$, and $10 \mathrm{~mm}$ thickness. The circular edge notch has a radius (r) of $1.5 \mathrm{~mm}$, and propagated sharp crack length (a) of $6 \mathrm{~mm}$. The initial hole at the crack tip has a radius $(\mathrm{R})$ of $1 \mathrm{~mm}$ the model being studied is a uniform, homogeneous plate symmetrical about horizontal axes in both geometry and loading. Finite element elastic-plastic analysis is done for plane stress conditions. Element type, (PLANE82) is chosen. Nonlinear static analysis is executed then optimization of hole shape is executed. Optimum hole shape is that produces minimum stresses and strain values without decreasing the nominal area of the model is obtained, as illustrated in the following Table 1 for half hole where $P$ for points, $R$ distance from between hole center and each point, and $\theta$ is the angle between specimen symmetry

\begin{tabular}{|c|c|c|c|c|c|c|c|c|}
\hline$P$ & I & 2 & 3 & 4 & 5 & 6 & 7 & $N_{N=}^{N}$ \\
\hline$\theta^{c}$ & 0.903 & 0.9836 & 1.1162 & 0.9767 & 0.9689 & 0.9802 & 1.0006 & \\
\hline & 0 & 300 & 600 & 900 & 1200 & 1500 & 1780 & \\
\hline
\end{tabular}
line and the point.
Determining plastic zone size at crack tip and at optimum hole

Theoretically, stress at the crack tip under plane tensile stress is infinite for a perfect elastic material. However, for most materials, the region around the crack tip yields and plastic deformation zone appears. Although calculating the precise shape of this zone is complicated, its size in the direction of crack propagation can be approximated easily. ${ }^{3}$ In the earlier calculation plastic zone size was calculated for $\theta=0$ (Along the crack plane), the plastic zone shape will be quite different when all angles are considered as illustrated in
Figure 1. Plasticity is based on Von Mises² According to Von Mises yield criteria the plastic zone shape could be estimated as

$$
r_{p}=\frac{1}{4 \pi}\left(\frac{K_{I}}{\sigma_{Y}}\right)^{2}\left(1+1.5 \sin ^{2} \theta+\cos \theta\right) \text {. Where } \mathrm{r} \_\mathrm{p} \text { is the plastic zone }
$$

radius, $\mathrm{K}_{-} \mathrm{I}$ the stress intensity factor is for edge crack specimen, $\sigma \_\mathrm{y}$ is the yield stress, $\Theta$ is the angle between crack surface and plastic zone boundary. Two identical rectangular blocks are studied, one with propagated sharp crack length, a, and the other with repaired crack by drilling a hole with optimum shape at its tip. Finite element 
analysis is done for both block as illustrated in Figure 1. Plastic zone at crack tip is calculated using VonMises yielding criteria. Using MATLAB software, plastic zone can be drawn as illustrated in Figure 2 the natures of plastic zones obtained in this analysis are in good agreement with the theoretical plastic zone shapes presented in literature. ${ }^{3}$ To compare between plastic zone size and optimum hola size; polar coordinate of plastic zone was calculated as illustrated in Figure 3. Then both of them were tabulated as indicated in Table 2. Where $r$ is the distance from crack tip to the outer surface of plastic zone around the crack tip, and $\mathrm{R}$ is the distance from hole center to the outer surface of the hole. By using ANSYS software both of optimum hole and plastic zone is drawn with respect to the same origin in order to compare between them, as illustrated in Figure 4. Figure 4 indicates two important results:

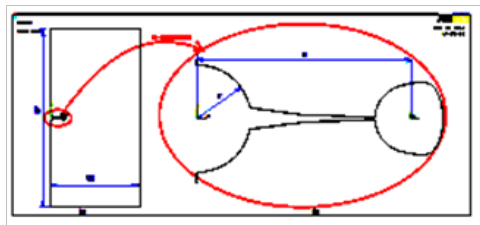

(a)

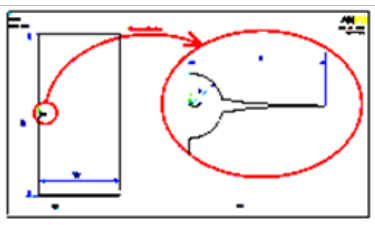

(b)
Figure I (a) Model with non repaired crack (b) Model with a repaired crack using optimum hole.

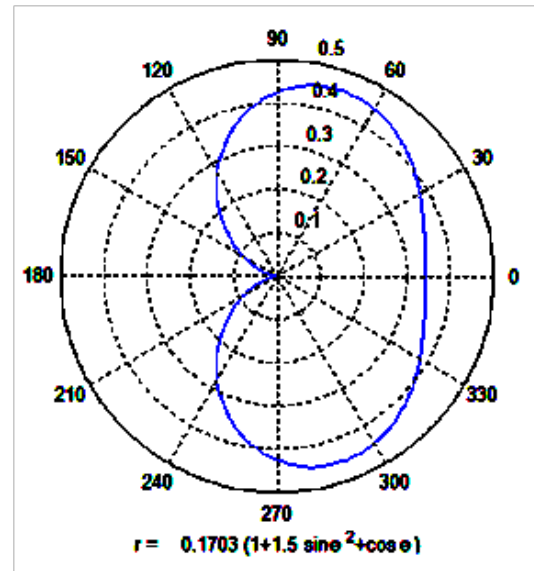

Figure 2 Plastic zone size.

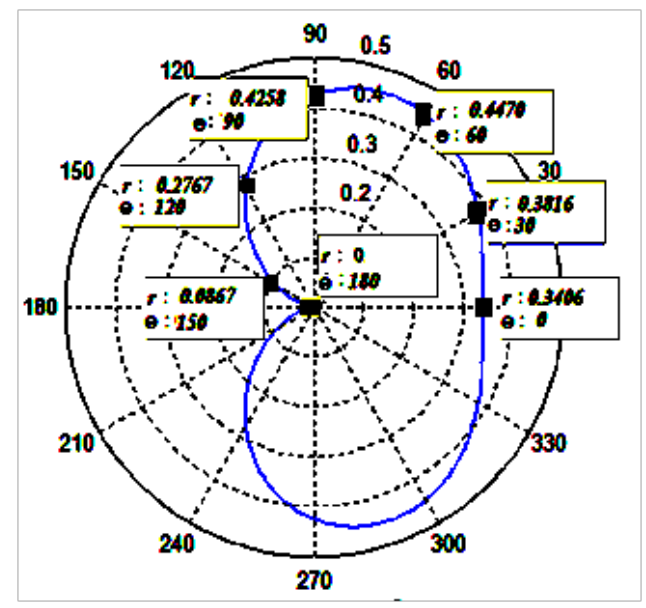

Figure 3 Polar coordinate of plastic zone.

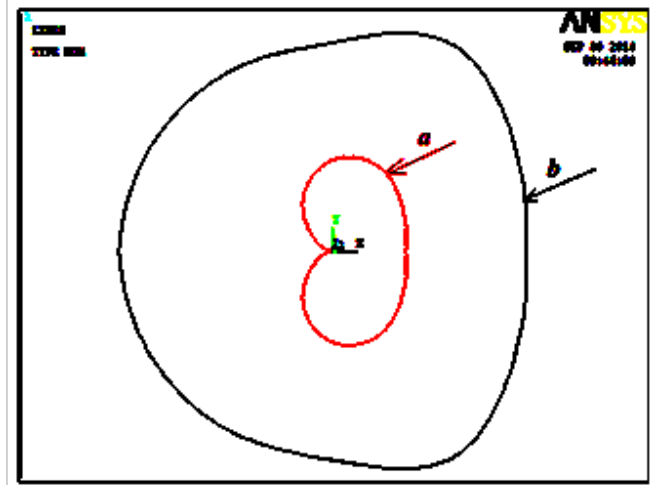

Figure 4 Similarity between optimum hole shape and plastic zone shape.

Table 2 Plastic zone and optimum hole polar coordinates

\begin{tabular}{llllllll}
\hline$\theta$ & 0 & 30 & 60 & 90 & 120 & 150 & 180 \\
$r$ & 0.3406 & 0.3816 & 0.447 & 0.4258 & 0.2767 & 0.0867 & 0 \\
$R$ & 0.903 & 0.9836 & 1.1162 & 0.9767 & 0.9689 & 0.9802 & 1.0006 \\
\hline
\end{tabular}

First: the similarity of the optimum hole shape to the plastic zone shape,

Second: as illustrated by all researchers studied stop-hole method stop-hole were much larger than the cyclic.

Plastic zones which followed the original fatigue crack tips to ensure that they did remove all the damaged material ahead of the cracks and, consequently, that the material at the resulting notch root can be treated as virgin. The optimum hole shape size would be sufficient to remove almost all of the plastically deformed particles ahead of the crack tip and leave the material in virgin stat. To prove that almost all of plastic deformed particle around crack tip will be removed by boring optimum hole, the stress results for cracked model were obtained as illustrated in Figure 5. In Figure 5(a) the tip of material elastic zone along $\mathrm{x}$ axis (crack growth direction) deformation began at equivalent stress nearly equal to $141 \mathrm{MPa}$ as illustrated by white color contour in Figure 5(a). In Figure 5(b) the same results with the same color map are plotted as a curve of $\sigma \_$yy (where $\sigma$ yy is the axial stress) stress versus distance behind crack tip. At distance equal to $0.9030 \mathrm{~mm}$ (which corresponding to optimum hole polar coordinate R1) most of area under stress curve had been removed and the stress reached elastic zone area (white color), that denotes that the material returned to its virgin stat.
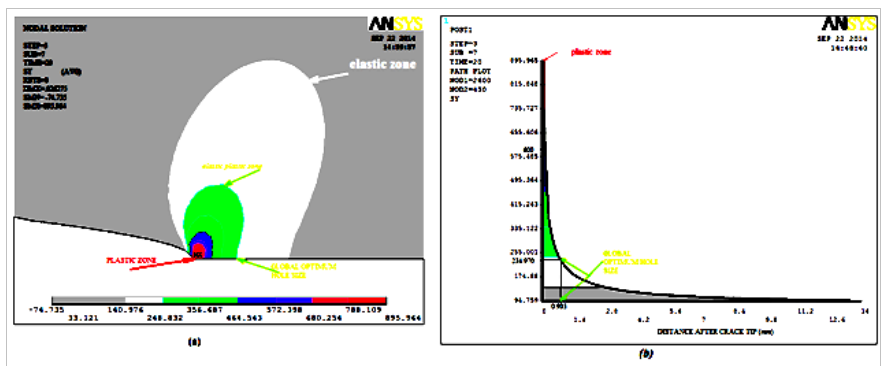

Figure 5 Stress distribution for unrepaired crack. (a) ${ }^{\sigma}$ yy Stress contour and plastic zone shape (b) Curve of stress versus distance from crack tip to the unmatched model side. 


\section{Conclusion}

The crack tip plastic zone refers to the crack forward plastic zone. This is the region of yielded material ahead of the crack tip at maximum load. The reverse plastic zone is the region of material ahead of the crack tip that yields in compression at the minimum load. The plastic zone size ahead of the crack tip is of crucial importance in this study as it is hypothesized that the hole should be drilled to remove most of this zone. This will leave the rest of the material in elastic state and eliminate any predefined slip planes of dislocation. Global optimum whole shape can remove almost all deformed particle in component material.

\section{Acknowledgments}

No acknowledgement.

\section{Conflicts of interest}

Author declares that there is no conflict.

\section{References}

1. Irwin GR. Fracture Mechanics. Structural Mechanics. 1960.

2. Von Mises. Mechanics of solid bodies in the plastically-deformable state. Math Phys. 1913;1:582-592.

3. FA McClintock, GR Irwin. Plastic aspects of fracture mechanics, fracture toughness testing and its applications. American Society for Testing and Materials. 1965;381:84-113.

4. Ragab. On blunting the crack tip through hole-drilling. Alexandria Engineering Journal. 2003;42(3):347-355.

5. Rahgozar R. Finite Element Analysis of Plasticity- Induced Fatigue Crack Closure with Singular Element. American Journal of Applied Sciences. 2007;4(6):357-361. 